\title{
Effect of circular dances on quality of life of intestinal ostomized persons: A randomized controlled trial
}

\author{
Cristilene Akiko Kimura*1, Dirce Bellezi Guilhem ${ }^{2}$, Breno Silva de Abreu ${ }^{3}$, Rodrigo Marques da Silva ${ }^{1}$, Karina Ribeiro \\ Modesto ${ }^{3}$ \\ ${ }^{1}$ Nursing Department, Faculdade de Ciências e Educação Sena Aires, Valparaíso de Goiás, Brazil \\ ${ }^{2}$ Health Sciences Department, Universidade de Brasilia, Brasilia, Brazil \\ ${ }^{3}$ Pharmacy Department, Faculdade de Ciências e Educação Sena Aires, Valparaíso de Goiás, Brazil
}

Received: July 19, 2019

DOI: $10.5430 /$ jnep.v10n4p36
Accepted: September 15, 2019 Online Published: December 23, 2019

URL: https://doi.org/10.5430/jnep.v10n4p36

\begin{abstract}
Background and objective: Circular dances have several benefits to the physical and mental health status of patients. few investigations analyze the effect of dances on the Quality of Life of intestinal ostomized patients. We assessed the effect of circular dances on quality of life of intestinal ostomized persons.

Methods: This is a randomized controlled trial conducted with 73 individuals were randomly clustered in two major groups: I) Controls to which the standard practices were applied and II) Cases to which all the standard procedures were applied but added the practices of circular dance. The Quality of life (QoL) score were assessed by the validated questionnaire COH-QOL-OQ.

Results: No significant differences between the domains' average scores before the intervention. The correlation between the average scores has decreased on the experimental group after the intervention suggesting that the circular dances managed to improve QoL scores.

Conclusions: Circular dance practices on ostomized individuals contributes to a better QoL and represents an improvement on nursing care quality. Furthermore, the permanent review of nursing practices contributes significantly to the integral health care and better nursing services delivery to the ostomized individuals. Findings confirm the efficacy of circular dances to improve de Quality of Life of intestinal ostomized persons.
\end{abstract}

Key Words: Comprehensive health care, Health policy, Nursing, Ostomy, Quality of life, Dance therapy

\section{INTRODUCTION}

The World Health Organization defines quality of life (QoL) as individual's perception of their position in life regarding: the culture and system of values in which they live; and their objectives, expectations, patterns and concerns. ${ }^{[1]}$ From this point of view, QoL is a subjective concept that impacts aspects, essential to a person's life.

Keeping physical integrity is important for the individual's well-being and social surrounding. The rupture of this integrity - what happens when an intestinal ostomy is mademay cause physical, psychological, spiritual and social problems. ${ }^{[2,3]}$ In this context, patients with intestinal ostomy need specialized healthcare in order to promote autonomy and QoL for themselves, their familiars and caregivers. ${ }^{[4,5]}$ Also, policies and nursing practices are required to increase the chances of rehabilitation and social reinsertion, what

\footnotetext{
*Correspondence: Cristilene Akiko Kimura; Email: cris.akiko7@ gmail.com; Address: Nursing Department, Faculdade de Ciências e Educação Sena Aires, Valparaíso de Goiás, Brazil.
} 
implies on these patients' QoL.

Nurses are responsible for identifying preventive actions, addressing patients' needs and establishing healthcare priorities based on theoretical approaches as well as planning and evaluating the patient progress through their recovery process. Additionally, the production, development and assessment of health technologies, education and ethics need to be considered to deliver an integral healthcare for intestinal ostomized patients and, consequently, improve their QoL. ${ }^{[6]}$

New actions that complement the traditional healthcare model for ostomized people-may facilitate the physical adaptation and social insertion of these patients. One alternative is the circular dances that aims to stimulate the individuals' body in a harmonic way, considering all their emotions and physiological status. An additional benefit is the strengthening of motor skills and self-knowledge. ${ }^{[7-9]}$ These dances are an artistic expression that has always been present in the history of humanity - birth, marriage, planting, harvesting, glove arrival, spring, death - and reflected in the need for communion, participation and unity among people. ${ }^{[7-9]}$ Then, circular dances are a kind of physical activity that may have a positive effect for relieving pain-related symptoms.

Circular dances are capable of improving the mental and physical arrangement, flexibility, posture, strength and muscular resistance and body conscience, relieving pain and tensions and, so, producing well-being for individuals. ${ }^{[8-10]}$ They also play an important role on mental and emotional components once dancing positively affect attention, memory, cognition, language, as well as the self-expression, socialization and relieving feelings of loneliness and social isolation. ${ }^{[9-12]}$

Although investigations on the benefits of dances in several populations are available, those about the effect of circular dances on the QoL are limited After assessing the effect of circular dances among 35 mastectomized Brazilian women (11 in the experimental group; 24 in the control group), the QoL in the psychological domain significantly increased in the experimental group after the intervention. ${ }^{[13]}$ Investigation conducted in Campinas (SP), Brazil with 10 women (Aged between 25-54 years) found an improvement in perceived body image, religiosity and QoL related to the circular dances. ${ }^{[14]}$ In addition, research with 80 women diagnosed with fibromyalgia found significant effect of a 16 week belly dancingregime on pain relief, functional capacity, self-image and QoL in the experimental group compared with the control one. ${ }^{[15]}$ Other research with 31 cancers survivor's women from Alabama (United States of America) verified that physical activity, mental component of QoL, vitality and trust were significantly affected for those who practiced ballroom dances. ${ }^{[16]}$

The purpose of this study was to investigate the effect of circular dances on the quality of life of intestinal ostomized patients.

\section{METHOD}

\subsection{Design}

A randomized and controlled clinical trial was conducted in the Outpatient Healthcare Program for ostomized persons, in Federal District, Brazil.

\subsection{Participants and Sampling}

Data were gathered from patients registered in the Outpatient Healthcare Program for ostomized persons- that pertains to the Health Department of the Federal District, Brazil. Men and women, aged 18 and over, with colostomies, nonpracticing of any dances or physical activities and underwent to the colostomy procedure more than 12 months before the data collection were included in the sample. In addition, we only selected those patients able to understand the intervention and to attend all program meetings. Individuals under 18, pregnant women, infants, bedridden, physical deficient persons and those with medical constraint to physical activities or presenting any physiological condition that restricted to attend the Practices of Circular Dances (PCD) were excluded from the study.

We considered four factors to determine the sample size: physical space of the health facilities; socioeconomic status; possible resistance to social interactions; and difficulties for public transportation. Even the transportation costs were covered for the researchers, the socioeconomic status of participants may impact on adherence to the intervention, specially due to the low income status. Besides, patients may avoid to be exposed to public transportation and social interactions due to the changes in self-image perception produced for the ostomy and the difficulties to handle with it in buses and trains. So, the analysis of health facilities allowed an amount of 100 individuals per session of PCD, but after considering the other factors, we stipulated 80 subjects for PDC sessions.

Before the intervention, the sociodemographic and clinical data were assessed in both groups(control and experimental). General QoL and its dimensions were evaluated in two time points- before and after the PDC sessions. Figure 1 shows the flow chart of participants' random allocation in control and experimental groups.

Participants were included in the study trough the intentional non-probabilistic sampling, i.e., according to the availability of patients during the data gathering. After recruiting the 
sample, we randomly allocated the patients in control $(\mathrm{n}=$ $40)$ and experimental $(n=40)$ groups, observing the placement rate of $1: 1$. The control group received the outpatient healthcare routinely delivered to ostomized persons. These procedures and the Practice of Circular Dances were applied to the experimental groups.
The randomization was done by an external heath professional, unaware of the sample features and interventions performed. Then, patients were randomly assigned to the groups trough a simple randomization procedure, performed in the Microsoft Excel (Office Package) with the function "Random". Thus, a list containing the subjects of each group was produced and sent to the researchers.

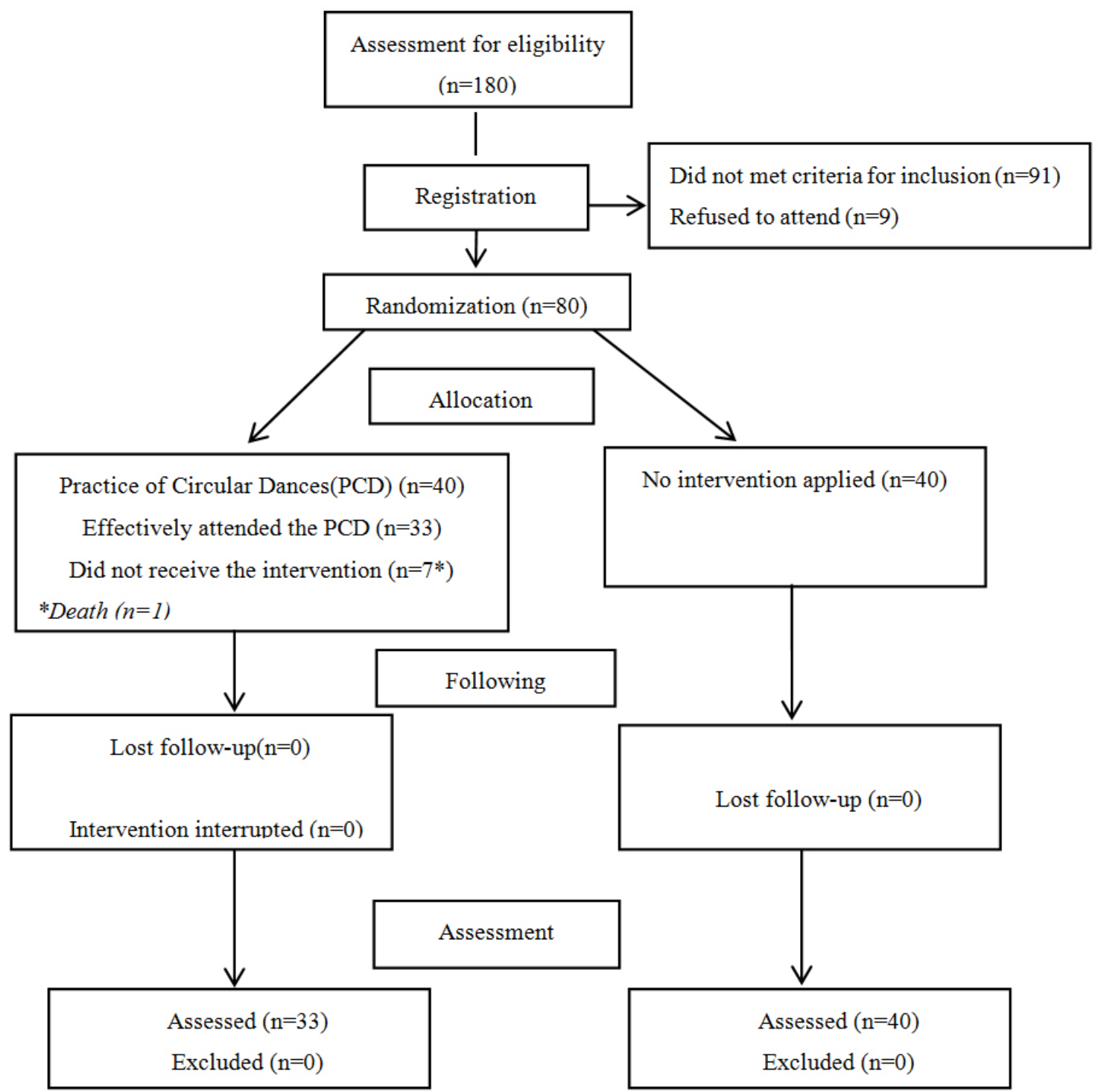

Figure 1. Flowchart of participants' random allocation in control and experimental groups

\subsection{Procedure}

The PCD was applied from February to June 2016. This intervention is based on traditional procedures from tribal cultures who seek for collective harmonization trough body movements guided by rhythmic songs. Also, participants are organized in a circle of $20 \mathrm{~cm}$ diameter, allowing in visualizing of each other throughout the dance. This interaction among participants integrates them each other, and provides a dissociation of individuals from the pathologic reality in which they are inserted.

The PCD was conducted with the experimental group for 12 consecutive weeks, twice a week, and lasted 60 minutes each time. The dances' steps- including gestures, movements and rhythms- were tough to the participants for a professional expert in PCD before starting the intervention in order to 
ensure a syntonic execution of the dance. All sessions were observed and recorded for researchers with audiovisual devices. After finishing the PCD in the experimental group, this intervention was applied in the control group.

\subsection{Instruments}

The following demographic variables were gathered: gender, marital status, familiar conviviality, religion, religious practice, education level, work status (working; retired; sick leaving), family income. The clinical data included cause of the intestinal ostomy, time of individuals live with the ostomy, type of ostomy, use of irrigation system and comorbidities. Additionally, we collected information about the follow-up of health services and the delivery of collector equipment by health professionals.

The COH-QOL-OQ was developed through the updating and expansion, in 1980, of the City of Hope - Quality of Life - Colostomy patients. ${ }^{[13]}$ The COH-QOL-OQ aims to assess the QoL of ostomized patients and is complained for 43 items distributed in four domains, as follows: Physical Well-Being(PhWB)- items 1 to 11; Psychological Well-Being (PsWB)- items 12 to 24; Social Well-being(SoWB)- items 26 to 36; and Spiritual Well-being (SpWB)-items 37 to 43. The responses are described in 10-point Likert scale, ranging from 0 (bad QoL) to 10 (excellent QoL). ${ }^{[14,15]}$

Patient scores were summed and divided for the number of items that comprise the referred domain, resulting in a mean score for each domain. The total score of QoL was obtained summing the values marked by individuals in all items of COH-QOL-OQ and dividing them for 43. Additionally, items 1 to $12 ; 15 ; 18$ and $19 ; 22$ to $30 ; 32$ to 34 ; and 37 have reverse scale. So, the scores were reversed before the data analysis.

\subsection{Data analysis}

Data were analyzed in the Statistical Package for Social Sciences(SPSS), version 20.0. Mean, standard-deviation, minimum and maximum were used for continuous variables. The $t$ Test, for parametric data, and the Mann-Whitney test, for non-parametric data, was used to compare the score of QoL. The Tukey Test (for multiples comparisons) and the ANOVA model were applied to compare the scores of QoL (domains and general scores) between the experimental and control groups. $p$ values $<.05$ were taken as statically significant.

\subsection{Ethical aspects}

Participants signed the Term of Free and Informed Consent Which explained the study and their rights. The Ethical
Committee at Brasilia University and the Health Secretary approved the research project under the protocols number 46323815.2.0000.0030 and 46323815.2.3001.5553, respectively.

\section{RESUlts}

From the 180 persons eligible for study, 80 were included in the investigation (40 in the control group and 40 in the experimental group. The flowchart of participants selection in details is described in Figure 1. Although 180 patients were eligible, 100 were excluded during the registrations in the healthcare service, resting 80 patients for the randomization process. Then, $50 \%$ of this amount were allocated in each group, but seven patients were removed from the intervention group. So, as final sample, 33 patients get the intervention and 40 were recruited for the control group. Table 1 shows the Sociodemographic features of experimental e control groups.

We found, in both groups, a prevalence of females, married, aged between 51 and 60 years, with familiar conviviality and who completed elementary school. They are Catholic or evangelic, who actively practice their religion, are retired or on sick leave, and receive between one and three minimum wages. Table 2 describe the Clinical features of experimental e control groups.

As shown in the Table 2, most of patients from both groups: are non smokers, do not have type 2 diabetes; don't use the Irrigation System; have definitive ostomies; live with the ostomy for $>12$ months and $\leq 60$ months; received clinical following and the collector equipment. However, a positive diagnostic for Arterial Hypertension was only predominant in the experimental group. Table 3 shows the Distribution of patients according to the cause of ostomy in experimental and control groups.

As presented in the Table 3, the two groups reported the Colorectal Cancer (Experimental: 51.5\%; Control: 60\%) as the main cause of ostomy. Table 4 shows the Mean scores of QoL's domains according to the COH-QOL-OQ for experimental and control groups in the first time-point.

Results presented in Table 4 attested that the mean scores of QoL' domains did not differ between the groups before the intervention, what confirm that the samples used in each group are homogeneous $(p>.05)$. Also, the two groups presented higher QoL in the Spiritual Well-being and Social Well-being domains. Table 5 shows the mean scores of QoL's domains according to the COH-QOL-OQ for experimental and control groups in the last time-point. 
Table 1. Sociodemographic features of experimental e control groups. Brasília, DF, Brazil, 2016

\begin{tabular}{|c|c|c|c|c|}
\hline \multirow{2}{*}{ Variables } & \multicolumn{2}{|c|}{ Experimental Group } & \multicolumn{2}{|c|}{ Control Group } \\
\hline & $\mathbf{N}$ & $\%$ & $\mathbf{N}$ & $\%$ \\
\hline \multicolumn{5}{|l|}{ Gender } \\
\hline Female & 20 & 61 & 24 & 60 \\
\hline Male & 13 & 39 & 16 & 40 \\
\hline Total & 33 & 100 & 40 & 100 \\
\hline \multicolumn{5}{|l|}{ Age range } \\
\hline 20------------------30 & 3 & 9.1 & 4 & 10 \\
\hline 31-------------------40 & 1 & 3.0 & 3 & 7.5 \\
\hline 41------------------50 & 5 & 15.2 & 5 & 12.5 \\
\hline 51--------------------60 & 13 & 39.4 & 13 & 32.5 \\
\hline 61-----------------70 & 10 & 30.3 & 10 & 25 \\
\hline 71-------------------80 & 1 & 3.0 & 5 & 12.5 \\
\hline 81-------------------90 & 0 & 0 & 0 & 0 \\
\hline Total & 33 & 100 & 40 & 100 \\
\hline \multicolumn{5}{|l|}{ Religion } \\
\hline Catholic & 21 & 63.7 & 26 & 65 \\
\hline Evangelic & 10 & 30.3 & 11 & 27.5 \\
\hline Spiritist & 1 & 3.0 & 3 & 7.5 \\
\hline Others & 1 & 3.0 & 0 & 0 \\
\hline Total & 33 & 100 & 40 & 100 \\
\hline \multicolumn{5}{|l|}{ Religious Practice } \\
\hline Yes & 28 & 84.8 & 31 & 77.5 \\
\hline No & 5 & 15.2 & 9 & 22.5 \\
\hline Total & 33 & 100 & 40 & 100 \\
\hline \multicolumn{5}{|l|}{ Marital Status } \\
\hline Married & 16 & 48.5 & 21 & 52.5 \\
\hline Stable Union & 3 & 9.1 & 10 & 25 \\
\hline Divorced & 3 & 9.1 & 2 & 5 \\
\hline Widowed & 4 & 12.1 & 2 & 5 \\
\hline Single & 7 & 21.2 & 5 & 12.5 \\
\hline Total & 33 & 100 & 40 & 100 \\
\hline \multicolumn{5}{|l|}{ Familiar Conviviality } \\
\hline With familiar conviviality & 29 & 87.8 & 38 & 95 \\
\hline Without familiar conviviality & 4 & 12.2 & 2 & 5 \\
\hline Total & 33 & 100 & 40 & 100 \\
\hline \multicolumn{5}{|l|}{ Education Level } \\
\hline No formal education to Basic School & 24 & 72.7 & 31 & 77.5 \\
\hline High School & 9 & 27.3 & 8 & 20 \\
\hline College & 0 & 0 & 1 & 2.5 \\
\hline Total & 33 & 100 & 40 & 100 \\
\hline \multicolumn{5}{|l|}{ Work Status } \\
\hline Retired & 12 & 36.4 & 21 & 52.5 \\
\hline Sick leaved & 12 & 36.4 & 11 & 27.5 \\
\hline Working & 4 & 12.1 & 1 & 2.5 \\
\hline Unemployed & 5 & 15.1 & 7 & 17.5 \\
\hline Total & 33 & 100 & 40 & 100 \\
\hline \multicolumn{5}{|l|}{ Familiar Income (MW*) } \\
\hline$<1$ a $3 \mathrm{MW}$ & 29 & 87.9 & 32 & 80 \\
\hline 4 a $5 \mathrm{MW}$ & 3 & 9 & 5 & 12.5 \\
\hline$\geq 6 \mathrm{MW}$ & 1 & 3.1 & 3 & 7.5 \\
\hline Total & 33 & 100 & 40 & 100 \\
\hline
\end{tabular}

*Brazilian Minimum Wage in 2016: R\$ 880.00 (About USD 271.6). 
Table 2. Clinical features of experimental e control groups. Brasília, DF, Brazil, 2016

\begin{tabular}{|c|c|c|c|c|}
\hline \multirow{2}{*}{ Variable } & \multicolumn{2}{|c|}{ Experimental Group } & \multicolumn{2}{|c|}{ Control Group } \\
\hline & $\mathbf{N}$ & $\%$ & $\mathbf{N}$ & $\%$ \\
\hline \multicolumn{5}{|l|}{ Time of Ostomized (in months) } \\
\hline$>12$ months and $\leq 60$ months & 27 & 81.9 & 30 & 75 \\
\hline$>60$ months & 6 & 18.1 & 10 & 25 \\
\hline Total & 33 & 100 & 40 & 100 \\
\hline \multicolumn{5}{|l|}{ Type of Ostomy } \\
\hline Definitive & 20 & 60.6 & 22 & 55 \\
\hline Temporary & 13 & 39.4 & 18 & 45 \\
\hline Total & 33 & 100 & 40 & 100 \\
\hline \multicolumn{5}{|l|}{ Use of Irrigation System } \\
\hline Yes & 0 & 0 & 1 & 2.5 \\
\hline No & 33 & 100 & 39 & 97.5 \\
\hline Total & 33 & 100 & 40 & 100 \\
\hline \multicolumn{5}{|c|}{ Comorbidities - Diabetes Mellitus } \\
\hline Yes & 10 & 30.3 & 8 & 20 \\
\hline No & 23 & 69.7 & 32 & 80 \\
\hline Total & 33 & 100 & 40 & 100 \\
\hline \multicolumn{5}{|c|}{ Comorbidities - Arterial Hypertension } \\
\hline Yes & 18 & 54.6 & 16 & 40 \\
\hline No & 15 & 45.4 & 24 & 60 \\
\hline Total & 33 & 100 & 40 & 100 \\
\hline \multicolumn{5}{|l|}{ Smoking } \\
\hline Yes & 11 & 33.3 & 17 & 51.5 \\
\hline No & 22 & 66.7 & 23 & 48.5 \\
\hline Total & 33 & 100 & 40 & 100 \\
\hline \multicolumn{5}{|l|}{ Clinical Following } \\
\hline Yes & 31 & 94 & 36 & 90 \\
\hline No & 2 & 6 & 4 & 10 \\
\hline Total & 33 & 100 & 40 & 100 \\
\hline \multicolumn{5}{|l|}{ Collector equipment receipt } \\
\hline Yes & 32 & 96.9 & 39 & 97.5 \\
\hline No & 1 & 3.1 & 1 & 2.5 \\
\hline Total & 33 & 100 & 40 & 100 \\
\hline
\end{tabular}

Table 3. Distribution of patients according to the cause of ostomy in experimental and control groups, Brasília, DF, Brazil, 2016

\begin{tabular}{|c|c|c|c|c|}
\hline \multirow{2}{*}{ Cause of Ostomy } & \multicolumn{2}{|c|}{ Experimental Group } & \multicolumn{2}{|c|}{ Control Group } \\
\hline & $\mathbf{N}$ & $\%$ & $\mathbf{N}$ & $\%$ \\
\hline Colorectal Cancer & 17 & 51.5 & 24 & 60 \\
\hline Abdominal and perineal traumas & 5 & 15.1 & 6 & 15 \\
\hline Intestinal Inflammatory Disease & 3 & 9 & 5 & 12.5 \\
\hline Chagas Disease & 4 & 12.2 & 1 & 2.5 \\
\hline Diverticular Disease & 4 & 12.2 & 4 & 10 \\
\hline Fournier Syndrome & 0 & 0 & 0 & 0 \\
\hline Total & 33 & 100 & 40 & 100 \\
\hline
\end{tabular}


Table 4. Mean scores of QoL's domains according to the COH-QOL-OQ for experimental and control groups in the first time-point. Brasília, DF, Brazil, 2016

\begin{tabular}{|c|c|c|c|c|c|c|c|c|c|c|c|}
\hline \multirow{2}{*}{$\begin{array}{l}\text { Groups } \\
\text { Domain }\end{array}$} & \multicolumn{5}{|c|}{ Experimental Group (First time point) } & \multicolumn{5}{|c|}{ Control Group ( First time point) } & \multirow{2}{*}{$p$} \\
\hline & $\mathbf{N}$ & Mean & SD* & CI 9 & & $\mathbf{N}$ & Mean & SD & CI 9 & & \\
\hline PhWB & 33 & 5.5 & 1.8 & 4.88 & 6.11 & 40 & 4.78 & 2.24 & 4.08 & 6.11 & .60 \\
\hline PsWB & 33 & 5.6 & 1.7 & 5.01 & 6.18 & 40 & 5.18 & 1.64 & 4.67 & 6.18 & .75 \\
\hline SoWB & 33 & 5.4 & 1.5 & 4.88 & 5.91 & 40 & 4.55 & 1.72 & 4.01 & 5.91 & .24 \\
\hline SpW & 33 & 7.1 & 1.8 & 6.48 & 7.71 & 40 & 7.35 & 1.70 & 6.82 & 7.71 & .42 \\
\hline
\end{tabular}

*Standard-deviation; PhWB: Physical Well-Being; PsWB: Psychological Well-Being; SoWB: Social Well-being; SpWB: Spiritual Well-being.

Table 5. Mean scores of QoL's domains according to the COH-QOL-OQ for experimental and control groups in the last time-point. Brasília, DF, Brazil, 2016

\begin{tabular}{|c|c|c|c|c|c|c|c|c|c|c|c|}
\hline \multirow{3}{*}{$\begin{array}{l}\text { Groups } \\
\text { Domain } \\
\text { PhWB }\end{array}$} & \multicolumn{5}{|c|}{ Experimental Group (Last time point ) } & \multicolumn{5}{|c|}{ Control Group ( Last time point ) } & \multirow{2}{*}{$p$} \\
\hline & \multirow{2}{*}{$\begin{array}{l}\mathbf{N} \\
33\end{array}$} & \multirow{2}{*}{$\begin{array}{l}\text { Mean } \\
6.7\end{array}$} & \multirow{2}{*}{$\begin{array}{l}\text { SD* } \\
1.6\end{array}$} & \multicolumn{2}{|c|}{ CI 95\% } & \multirow{2}{*}{$\begin{array}{l}\mathbf{N} \\
40\end{array}$} & \multirow{2}{*}{$\begin{array}{l}\text { Mean } \\
4.88\end{array}$} & \multirow{2}{*}{$\begin{array}{l}\text { SD } \\
2.34\end{array}$} & \multicolumn{2}{|c|}{ CI 95\% } & \\
\hline & & & & 6.18 & 7.29 & & & & 4.06 & 5.52 & $<.001$ \\
\hline PsWB & 33 & 6.4 & 1.0 & 6.11 & 6.79 & 40 & 5.06 & 1.52 & 4.61 & 5.81 & $<.001$ \\
\hline SoWB & 33 & 5.6 & 1.3 & 4.92 & 5.77 & 40 & 4.35 & 1.72 & 4.13 & 5.16 & $<.031$ \\
\hline SpW & 33 & 8.2 & 1.8 & 7.81 & 8.63 & 40 & 7.41 & 1.70 & 6.87 & 7.79 & $<.001$ \\
\hline
\end{tabular}

*Standard-deviation; PhWB: Physical Well-Being; PsWB: Psychological Well-Being; SoWB: Social Well-being; SpWB: Spiritual Well-being.

\section{Discussion}

Sociodemographic and clinical characteristics of control and experimental groups The mean age was about 50 years in both groups with colorectal cancer rates higher in those patients over the age of 50. These results are similar to those described in the scientific literature where researchers found more than $90 \%$ of colorectal cancer in individuals over 50 years old. ${ }^{[2,3,16]}$

In the experimental group, we found that $48.5 \%$ of patients were married and $9.1 \%$ had a stable union. In the control group, the results are similar: $52.5 \%$ of patients were married and $25 \%$ had a stable union. Also, in both groups, we found that ostomized persons who declare themselves as divorced, widowed or single show a lower quality of life for Physical well-being and Psychological well-being domains. Independently of the relationship quality, the condition of not being married is a risk factor for health. Thus, the divorced, widowed or single individuals may present a worse physical and mental health than the married patients. ${ }^{[4,17-20]}$ The familiar conviviality was prevalent in both groups, being a key factor for improving the quality of life once that family is perceived as a source of support, from where patients obtain help to deal with the ostomy and with their physical and emotional needs, being all connected for the affectivity bound..$^{[2,5,18,19]}$

Investigations highlight the relation between education level and QoL, being that persons with higher level of education present a better QoL. They also state that economic issues may affect the ostomized patients' QoL. ${ }^{[2,6,18-20]}$ In this re- search, however, a small part of the sample with a better level of education and higher income showed a higher QoL. For the variable work, in both groups, there was prevalence of retired patients who has a definitive ostomy. The ostomized person is considered a carrier of special need according to the Decree $n^{0} 5.296$ of 2 December 2004. ${ }^{[2,4,5]}$

Only one patient, from the control group, reported using the irrigation system. It arises the need of health professionals spread the irrigation technique and its related-knowledge for other health professionals and community. ${ }^{[2,6]}$ The use of irrigation is a method free of side effects that allows the intestinal control and benefit the social and parental relationships, increasing the ostomized persons' QoL. ${ }^{[2,6,20,21]}$

\subsection{Comparison of quality of life between the control and experimental groups}

In the pre-intervention assessment, we did not find significant differences between the control and experimental groups, being that both groups showed higher scores of QoL in Spiritual and Social well-being domains. When the QoL were assessed after the intervention, we observed a significant difference between the groups, being that the QoL was higher in the Experimental group when compared to the control. Finally, the comparison the groups before and after the intervention demonstrated a significant change in all QoL's domains after the intervention in the experimental group. It confirms the effectivity of the PCD for improving the QoL of intestinal ostomized patients. 
In this context, few investigations have shown positive results regarding the variables assessed in this study, with a good adherence of participants to the PCD. The arrangement in circle promotes the attention to the rhythm of the group as a whole; well as the improvement of subjective and positive states that help to deal with the ostomy, contributing to the therapy adherence. ${ }^{[7,22]}$

Researchers described the existence of values and feelings associated with the circular dance. They stand out that the practice of dancing in circle allows different ways of communication among the dancers; and represents an important rescue of traditions and movement, linking song and movement. ${ }^{[23,24]}$ Also, the circular dances stimulate the expression of individuals according to their needs, i.e., nobody is obligated to do something that don't desire. In addition, the fear of failing or incorrectly execute the dance steps are replaced for the feeling of satisfaction, associated with the overcoming and mutual support given for peers into the group. This kind of dance allows that people connect to themselves, making them respecting the body limitations and exploring potentialities. $^{[22,25]}$

Among the global benefits of circular dances is the favoring of the equilibrium center. The person starts to feel simultaneously their both hemi bodies, what improves their ability of perception, laterality and proprioception. Besides, other benefits include the gain of strength and muscular resistance; and the enhancement of motor control, contributing to the global motor coordination, balance and body conscience. ${ }^{[25,26]}$

The dance- trough the body movement and the change of self-perception- provides a relaxing that may relieve the pain, improves the physical well-being and reduces the number of sensible points and muscular contractures. ${ }^{[10,26]}$ Additionally, dancing may significantly contribute to improve sleep pattern; and relieve anxiety, muscular tension, and fatigue. ${ }^{[10,27,28]}$ In an investigation approaching the circular dances, the participants of PDCs became more willing to daily activities and work; and showed a better sleep quality. The same study also demonstrated that the physical activity provided for PDCs stimulate the body functions and, so, improve the locomotor system, what helps in the day-to-day demands. ${ }^{[22]}$ Thus, we confirm the effect of PDCs to improve the QoL of intestinal ostomized persons, with findings that are in consonance with the national and international scientific literatures.

\subsection{Limitations}

One of the limitation refers to the sample size that may restrict the inferences about the findings of study. Also, changes in the eligibility criteria, such as: minimum period of intestinal ostomy smaller than one year; and the inclusion of younger persons to the sample may limit generalized findings from this study. Given the small sample size the isolated effects of PCD may be better understood with a higher sample size, what was not possible in this study due to the costs, time and magnitude of the trial.

\subsection{Interpretation}

In general, results show the importance of adopting of integrative practices, such as the PCD for intestinal ostomized persons. This intervention had a significant effect on improving the general QoL and its domains: Physical Well-Being; Psychological Well-Being; Social Well-being; e Spiritual Well-being. No intervention-related damage was reported for patients.

\subsection{Generalization}

The findings suggest that PCD is an essential tool to assist patients improve their own health status. Findings from this study suggest that PCDs improve interpersonal relationships tallow for addressing problems/issues as they develop, and allow for the empowerment of the personal networks and the possibilities of solving adverse situations in partnership with the local community.

\section{Conclusion}

We verified that the PCDs may be considered an important method to promote and maintain a good health status in intestinal ostomized persons. Also, PCDs allows stimulating the integral body and mind perception trough the self-knowledge of competences that happens due to the socialization promoted for PCDs. The possibility of go hand in hand, and feel the harmony into de group trough the movement and rhythm solve boundaries.

The permanent training of health professionals, especially nurses, about issues related to the process of living with intestinal ostomies contribute significantly for an integral and qualified healthcare to these individuals. These trainings may also include the PCD's intervention in order to improve the patients' QoL in its all domains: Physical, Psychological, Social and Spiritual Well-being. In this perspective, the permanent education turned to the National Politics of Integrative and Complementary Practices (PNPIC) for health workers that deal with ostomized persons is essential to improve the quality of healthcare provided and the QoL of these people.

\section{CONFLicts OF INTEREST Disclosure}

This investigation is registered in the Clinical Trials Brazilian Registry (ReBEC) under the identifier RBR7VXCXM. The full trial protocol may be found in 
www.ensaiosclinicos.gov.br/rg/ with the title "Effects of circular dances on quality of life of intestinal ostomized persons: a randomized controlled trial". This project was granted for the Technological Center of Education Sena Aires and the recommendations of the Consolidated Standards of Reporting Trials (CONSORT) were followed to conduct the study and its reporting.

\section{REFERENCES}

[1] World Health Organization. The WHOQOL Group. Quality of life assessment (WHOQOL): position paper from the World Health Organization. Soc Sci Med. 1995; 41(10): 1403-9. https://doi.or g/10.1016/0277-9536(95) 00112-K

[2] Kimura CA, Kamada I, Jesus CAC, et al. Quality of Life of Colorectal Cancer Patients with Intestinal Stomas. Journal of Carcinogenesis \& Mutagenesis. 2014; (5): 1-7.

[3] Kimura CA, Kamada I, Guilhem DB. Quality of life in stomized oncological patients: an approach of integrality from Brazilian Unified Health System. Journal Coloproctol. 2016; 36 (1): 34-9. https://doi.org/10.1016/j.jcol.2015.12.003

[4] Kimura CA, Kamada I, Guilhem D, et al. Perception of sexual activities and the care process in ostomized women. Journal of Coloproctology. 2013; 33(3): 145-50. https://doi.org/10.1016/j . jcol.2013.05.004

[5] Martins LM, Sonobe HM, Vieira FS, et al. Rehabilitation of individuals with intestinal ostomy. British Journal of Nursing. 2015; 24(22): 4-11. PMid:26653721 https://doi .org/10.12968/bjon. 2015 .24.Sup22.S4

[6] Kimura CA, Guilhem DB, Kamada I, et al. Contribution of circular dance on quality of life on oncology patients bearing intestinal ostomy. J Med Oncl Ther. 2016; 1(2): 84-6.

[7] Behrends A, Müller S, Dziobek I. Moving in and out of synchrony: A concept for a new intervention fostering empathy through interactional movement and dance. The Arts in Psychotherapy. 2012; 39: 107-16. https://doi.org/10.1016/j . aip. 2012.02.003

[8] Kiepe MS, Stöckigt B, Keil T. Effects of dance therapy and ballroom dances on physical and mental illnesses: A systematic review. The Arts in Psychotherapy. 2012; 39: 404-11. https://doi.org/10.1 016/j.aip.2012.06.001

[9] Kimura CA, Guilhem DB, Kamada I, et al. Life quality for ostomized patients: a perspective in the health and nursing care process. Journal of Nursing Education and Practice. 2017; 7(4): 22-31. https://doi.org/10.5430/jnep.v7n4p22

[10] Baptista AS. Effectiveness of dance in patients with fibromyalgia: A randomised, single-blind, controlled study Clinical and Experimental Rheumatology. 2012; 30(74): 18-23.

[11] Wosien B. Dança um caminho para a totalidade/Bernhard Wosien; edição Maria- Gabriele Wosien; tradução Maria Leonor Rodenbach, Raphael de Haro junior. São Paulo: TRIOM, 2000.

[12] Aktas G, Ogce F. Dance as a therapy for cancer prevention. Asian Pacific Journal of Cancer Prevention. 2005; 6(3): 408-11.

[13] Gomboski G. Adaptação cultural e validação do city of hope - quality of life - ostomy questionnaire para a língua portuguesa no Brasil. 159 f. Dissertação (Mestrado em Enfermagem)-Escola de Enfermagem da Universidade de São Paulo. 2010.

[14] Grant M, et al. Revision and Psichometric Testing of the City of Hope Quality of Life Ostomy Questionaire, Quality of Life Research Journal. 2004; 13(8): 1445-57. PMid:15503840 https: //doi.org/10.1023/B:QURE.0000040784.65830.9f
[15] City Of Hope and Beckman Research Institute. Quality of life questionnaire for a patient with an ostomy. 2013. Available from: http://prc.coh.org/Ostomy-11.pdf

[16] Brasil Ministério da Saúde. Instituto Nacional do Câncer. Estimativa 2016: incidência de câncer no Brasil. Rio de Janeiro: INCA; 2015

[17] Censo Demográfico 2010. Características gerais da população, religião e pessoas com deficiência. Rio de Janeiro: IBGE, 2011. Acompanha 1 CD-ROM.

[18] Dabirian A, Yaghmaei F, Rassouli M, et al. Quality of life in ostomy patients: a qualitative study. Patient Preference and Adherence. 2011; 5(1): 1-5. PMid:21311696 https://doi.org/10.2147/PPA.S1 4508

[19] Fortes RC, Monteiro TMTC, Kimura CA. Quality of life from oncological patients with definitive and temporary colostomy. Journal of Coloproctology. 2012; 32(30): 253-9. https ://doi .org/10.159 0/S2237-93632012000300008

[20] Torres CRD, Andrade EMLR, Santos FM, et al. Quality of life of stomized people: an integrative review. Rev Enferm UFPI. 2015; 4(1): 117-22. https://doi.org/10.26694/reufpi.v4i1.2242

[21] Cesaretti IUR, Santos VLCG, Vianna LAC. Qualidade de vida de pessoas colostomizadas com e sem uso de métodos de controle intestinal. Rev Bras Enferm. 2010; 63(1): 16-21. PMid:20339749 https://doi.org/10.1590/S0034-71672010000100003

[22] Corazza DI. Influência de um programa sistematizado de danças circulares em aspectos psiconeuroimunológicos de idosos cuidadores de indivíduos com doença de Alzheimer. 149 f. Instituto de Biociências do Campus de Rio Claro, Universidade Estadual Paulista. 2014.

[23] Vankova H, Holmetova I, Machacova K, et al. The Effect of Dance on Depressive Symptoms in Nursing Home Residents. JAMDA. 2014; 1-6.

[24] Frison FS. Dança circular e qualidade de vida em mulheres mastectomizadas: um estudo piloto. Master Dissertation. Universidade Estadual de Campinas.

[25] Lee SY, Franchetti MK, Imanbayev A. Non-pharmacological prevention of major depression among community-dwelling older adults: A systematic review of the efficacy of psychotherapy interventions. Arch Gerontol Geriatr. 2012; (55): 522-9. PMid:22483200 https://doi .org/10.1016/j.archger.2012.03.003

[26] Mala A, Karkou V, Meekums B. Dance/Movement Therapy (D/MT) for depression: A scoping review. The Arts in Psychotherapy. 2012; 39: 287-95. https://doi.org/10.1016/j.aip. 2012.04.002

[27] Anaraki F, Vafaie M, Behboo R, et al. Quality of Life Outcomes in Patients Living with Stoma. Indian Journal of Palliative Care. 2016; 18(3): 176-80. PMid:23439841 https://doi.org/10.4103/09 73-1075. 105687

[28] Iqbal F, Kujan O, Bowley DM, et al. Quality of Life After Ostomy Surgery in Muslim Patients. J Wound Ostomy Continence Nurs. 2016; 43(4): 1-7. PMid:27196687 https ://doi.org/10.1097/WON . 00 00000000000235 